\title{
Comparison of Optimization Frameworks for the Design of a Multi-Energy Microgrid
}

\author{
Rémy Rigo-Mariani ${ }^{\mathrm{a}}$, Sean Ooi Chea Wae ${ }^{\mathrm{a}}$, Stefano Mazzoni ${ }^{\mathrm{a}}$, Alessandro Romagnoli ${ }^{\mathrm{a}, \mathrm{b}}$ \\ a Energy Research Institute at NTU (ERI@N), Nanyang Technological University, Singapore \\ ${ }^{\mathrm{b} S}$ School of Mechanical and Aerospace Engineer, Nanyang Technological University, Singapore
}

Abstract-The scope of the paper is to investigate different strategies for the design of a multi-energy system considered as a systemic optimization problem. The objective is to determine the best sizes of energy assets such as electrochemical and thermal storages, cogeneration units, solar generators and chillers. In these cases, the techno-economic optimization is a tradeoff between the operating costs and the capital expenditures in the form of integrated management and design of the system. The paper addresses the challenges of these optimization problems in two steps. The former implements generic piecewise linearization techniques based on non-linear models. That approach allows a significant reduction of the computational time for the management loop of the assets (i.e. optimal power dispatch). The latter takes into consideration the integration of that management loop in different architectures for optimal system planning. The main contribution of the paper toward filling the gap in the literature is to investigate a wide range of optimization frameworks - with bi-level optimizations (using both deterministic and evolutionary methods), Monte-Carlo simulations as well as a performant 'all-in-one' approach in which both sizing and controls are variables of a single mathematical problem formulation. Finally, a thorough results analysis highlights that the best solution tends to be the same whether the objective to optimize is the traditional net present value at the end of the system lifespan or the total yearly cost of ownership.

Key Words-Multi-energy, optimal planning, quadratic programming, mixed integer linear programming, evolutionary algorithms

\section{INTRODUCTION}

For decades now, power and energy systems have face a significant change of paradigm with the deployment of renewable energy resources, mainly motivated by the need to reduce the greenhouses gases emissions. The variability of the renewables during short and long term periods represents the main drawbacks of the adoption and integration of those resources, especially wind and solar. Indeed, their generated powers are not controllable and are subject to uncertainties that may endanger the secure operations of energy systems (e.g. power grid voltage/frequency stability). These shortfalls can be overcome while providing more flexibility from the endusers side where legacy utility system (and especially power systems) are vertically organized from heavy duty generators down to load points [1]. Thus, the concept of Decentralized Energy Resources (DER) emerged in the past twenty years. DER refer to smaller scale assets, typically below $1 \mathrm{MW}$, which are connected near the end-users. This equipment can refer to local generation (renewable or not), storage devices as well as controllable loads aggregated in microgrids [2]. Under this context, DER allow for easier penetration of renewables into its overall generation. Their closeness to the end-user further reduces the energy losses and allows the deferment of investments for grid expansion (e.g. lines, power transformers). 
Polygeneration technologies are important in DER as they have shown to increase the flexibility and the efficiency at the enduser point by taking advantage of the interaction between different energy carriers (e.g. electricity, gas, chilled water) [3]. The optimal design of so-called multi-energy microgrids has thus become a critical class of problems addressed in literature in the framework of renewable energy integration [4], [5]. In the parametric analysis with different microgrid components sizing proposed by [6] it was found that there is an evident correlation between PV sizing and battery capacitance. Above a certain threshold value, the battery pack becomes less convenient. The optimal planning framework proposed by [7], which performs the optimal structure configuration together with the energy management strategy for multi-energy systems using mixed integer linear programming (MILP), has shown to provide better economic and environmental performances compared to conventional centralized energy systems with no coupling of energy converters: the power loads are supplied by an outside power distribution network, heating loads are satisfied by electric heat pumps and cooling loads are met by electric chillers respectively. Similar studies by [8] and [9] in the design of distributed multi-energy systems point to the same conclusion. [10] adopted a multi-node modelling approach to include operational constraints to their electrical heating and cooling networks in order to more accurately evaluate the investment cost of multi-energy microgrids. A study by [11] also integrated the mobility (electric vehicles) and water desalination sector alongside power, cooling and gas when modelling an urban energy system. Their proposed stepwise increase in energy system integration to transit to a smart urban energy system showed significant socio-economic cost savings, reduction in $\mathrm{CO}_{2}$ emissions and lower primary energy supply. In addition, [12] and [13] have highlighted the $\mathrm{CO}_{2}$ emission reduction achieved when adopting multi-energy systems.

Mathematically, such planning problems refer to systemic optimization where the operation of a system is considered from the design phase. The fundamental idea is to attain a compromise between the cost of the assets (capital expenditures CAPEX) and the benefit resulting from their reduced operating expenses (OPEX) [4]. In terms of the optimization framework used, several architectures are usually implemented to replace the legacy approach, which consists of independently optimizing the design and the operation. Traditional methods may refer to Monte Carlo simulations with different sizes of the assets randomly generated and the operating expenses computed for each configuration. [15] proposes such an approach with the management of a microgrid linearly solved over a representative week and a hybrid analytical/random procedure that investigates different capacities. Bi-level procedures are especially popular and consist of two integrated optimization problems with an outer loop optimizing the design and an inner level that computes the operating expenses for the corresponding capacities. Evolutionary procedures are often favored to explore the space of possible sizes such as Genetic Algorithm (GA) [16] or Particle Swarm Optimization (PSO) [17]. In such frameworks, the main concern is that the computational time of the management loop should be short enough as it is computed several times for many different system configurations. Thus, the use of linearized approaches or (MILP is often preferred [16] [18] along with the estimation of the operating expenses on a limited set of representative days [17]. Other widely used approaches realize an 'all-in-one' optimization where both operating and sizing parameters of the considered system are variables of a single optimization problem. Typically, the convexity of the inner loop management problem allows the expression of its equivalent KKT conditions (Karush-Kuhn-Tucker) at the global optimum point [19]. The KKT equations are then integrated into an optimal sizing problem to find the best configuration with regards to the objectives [20]. Simpler methods do not require the use of KKT conditions and the operation of the system is entered as a set of objectives and constraints in the sizing problem. Linear and MILP solvers are used to avoid prohibitive computational times [21] [22]. The main advantage of such approaches is that they guarantee to return the global optimum of the considered problem, provided it can be expressed under a linear formulation.

The main objective of this paper is to propose a comprehensive comparison between the aforementioned optimization frameworks. The case study consists in a building-scale multi-energy system with both electrical and thermal loads. The objective is to 
determine the best sizes of energy assets such as electrochemical and thermal storages, a Combined Cooling, Heat and Power unit (CCHP), solar generators and chillers. The chiller plant, with its consumption of electricity and production of cooling power, is one of the units that links the electrical and thermal energy vectors. Furthermore, the exhaust gases from the engine supply the thermal compressor of an absorption chiller that provides additional cooling. As such, the CCHP unit also links the electricity and cooling generations. Although an integrated network can exploit the interdependency between multiple energy vectors, they come at the cost of increasing the complexity of the problem. The connectivity between both buses through the components thus has to be taken into account during the formulation of the problem. The main contributions of the paper are:

- A generic linearization for the operations of the gas engine and chiller plant based on nonlinear models of the equipment. The integration of the linearized model into a MILP problem for the optimal power/cooling dispatch.

- The investigation of diverse optimization framework to perform the optimal planning of the considered system (Monte Carlo Simulation, Bi-level optimizations and 'all-in-one' procedure)

- A comprehensive results analysis with a clustering of the obtained solution and their discrimination regarding different techno-economic criteria - operating expenses and capital expenditure, the Total Cost of Ownership (TCO) and Net Present Value (NPV). The results, in particular, show the equivalence of the best solution when optimizing the TCO of the system and its NPV at the end of the expected lifetime.

The paper is organized with section 2 describing the base case study and the typical models used to estimate the OPEX of the system, for a given set of capacities of the different assets (i.e. gas engine, solar generator, etc). Especially, that estimation lies in an economical power dispatch over a representative day aiming at minimizing the overall energy bill thanks to electricity and gas prices arbitrage. Then, section 3 focuses on the modified formulation of the dispatch problem in order to integrate the linearized formulation for the gas engine and chiller operations. Test runs are performed in order to estimate the deviations with the results obtained using the 'reference' models. The objective is to reduce the computational time for the estimation of the operating cost with the use of approximate models. Finally, section 4 integrates the OPEX estimation in an optimal planning of the system that performs a tradeoff between the operating cost and the capital expenditures. Especially, different optimization architectures are investigated and compared in terms of obtained solutions (i.e. optimal design) and computational times. A result analysis is performed with regards to different sets of criteria before the conclusions are drawn in section 5 .

Table 1: Nomenclature of the used symbols and acronyms

\begin{tabular}{|c|c|c|c|}
\hline Sets : & & $\underline{S O C^{b a t}}, \overline{S O C^{b a t}}$ & battery $\mathrm{min} / \mathrm{max}$ state of charge $(\%)$ \\
\hline$t \in T$ & set of time steps $(24 \mathrm{~h})$ & $\underline{S O C^{t s}}, \overline{S O C^{t s}}$ & $\min / \max$ state of charge of battery $t s(\%)$ \\
\hline$k \in K$ & set of piecewise linear (PWL) blocks & $S O C_{0}^{b a t}, S O C_{0}^{t s}$ & battery and thermal storage initial state of charge $(\%)$ \\
\hline$b_{k} \in B_{K}$ & set of breakpoints for PWL $(=K-1)$ & $m_{b_{k}}^{g e}, p_{b_{k}}^{g e}$ & gas engine PWL breakpoints $(\mathrm{kg} / \mathrm{s}, \mathrm{kW})$ \\
\hline Variables : & & $q_{b_{k}}^{c h}, p_{b_{k}}^{c h}$ & chiller PWL breakpoints $(\mathrm{RT}, \mathrm{kW})$ \\
\hline$P_{t}^{g d+}, P_{t}^{g d-}$ & grid import/export at time $t(\mathrm{~kW})$ & COPch & chiller plant coefficient of performance (-) \\
\hline$P_{t}^{g e}$ & gas engine power at time $t(\mathrm{~kW})$ & $C O P^{a b c h}$ & absorption chiller coefficient of performance (-) \\
\hline$M_{t}^{g e}$ & gas mass flow at time $t(\mathrm{~kg} / \mathrm{s})$ & $\beta_{p w h}^{a b c h}$ & absorption chiller power to waste heat ratio (RT/kW) \\
\hline$p_{k, t}^{g e}$ & gas engine power in block $k$ at time $t(\mathrm{~kW})$ & $\beta_{p c r}^{a b c h}$ & absorption chiller power per cooling ratio $(\mathrm{kW} / \mathrm{RT})$ \\
\hline$\alpha_{b_{k}, t}^{g e}$ & weight for gas engine breakpoint $b_{k}$ at time $t(-)$ & $\overline{P^{g d}}, \underline{P^{g d}}$ & grid contracted capacity import and max. export $(\mathrm{kW})$ \\
\hline$u_{t}^{g e}$ & gas engine on/off status at time $t\{0,1\}$ & Sizes : & \\
\hline$u_{k, t}^{g e}$ & gas engine status in PWL block $k$ at time $t\{0,1\}$ & $P_{R}^{p v}$ & solar generator rated power $(\mathrm{kWp})$ \\
\hline
\end{tabular}




\begin{tabular}{|c|c|c|c|}
\hline$P_{t}^{b a t+}, P_{t}^{b a t-}$ & battery charge/discharge at time $t(\mathrm{~kW})$ & $P_{R}^{g e}$ & gas engine rated power $(\mathrm{kW})$ \\
\hline$S O C_{t}^{b a t}$ & battery state of charge at time $t(\%)$ & $P_{R}^{b a t}, E_{R}^{b a t}$ & battery rated power and capacity $(\mathrm{kW}, \mathrm{kWh})$ \\
\hline$P_{t}^{c h}$ & chiller plant electrical load at time $t(\mathrm{~kW})$ & $Q_{R}^{t s}, E_{R}^{t s}$ & thermal storage rated power and capacity (RT, RTh) \\
\hline$\alpha_{b_{k}, t}^{c h}$ & weight for chiller breakpoint $b_{k}$ at time $t(-)$ & $Q_{R}^{c h}$ & chiller rated capacity (RT) \\
\hline$u_{t}^{c h}$ & chiller plant on/off status at time $t\{0,1\}$ & Acronyms : & \\
\hline$u_{k, t}^{c h}$ & chiller status in PWL block $k$ at time $t\{0,1\}$ & DER & Distributed Energy Resources \\
\hline$P_{t}^{a b c h}$ & absorption chiller electrical load at time $t(\mathrm{~kW})$ & OPEX & Operating Expenses \\
\hline$Q_{t}^{c h}$ & chiller plant cooling generation at time $t(\mathrm{RT})$ & CAPEX & Capital Expenditures \\
\hline$Q_{t}^{a b c h}$ & absorption chiller cooling generation at time $t$ (RT) & NPV & Net Present Value \\
\hline$Q_{t}^{t s^{+}}, Q_{t}^{t s-}$ & charging/discharging of thermal storage at time $t(\mathrm{RT})$ & TCO & Total Cost of Ownership \\
\hline$S O C_{t}^{t s}$ & Thermal storage state of charge at time $t(\%)$ & $\mathrm{CCHP}$ & Combined Cooling, Heat and Power unit \\
\hline Parameters : & & PWL & Piecewise Linearization \\
\hline$P_{t}^{l}$ & building electrical load at time $t(\mathrm{~kW})$ & MILP & Mixed Integer Linear Programming \\
\hline$P_{t}^{p v}$ & solar generation at time $t(\mathrm{~kW})$ & SQP & Sequential Quadratic Programming \\
\hline$Q_{t}^{l}$ & building thermal load at time $t$ (RT) & LHS & Latin Hypercube Sampling \\
\hline$\pi_{t}^{e}, \pi_{t}^{g}$ & electricity/gas prices at time $t(\$ / \mathrm{kWh}, \$ / \mathrm{kg})$ & GA & Genetic Algorithm \\
\hline$\eta^{\text {bat }}, \eta^{t s}$ & battery and thermal storage efficiencies (-) & PSO & Particle Swarm Optimization \\
\hline
\end{tabular}

\section{OPERATING COST ESTIMATION}

\subsection{Multi-Energy Systems}

The work presented in this paper falls under the framework of the Smart Multi-Energy System project, whose objective is the implementation of an energy management strategy at a building scale or for a cluster of buildings, demonstrating potential savings in costs and pollutant emissions. Indeed, this approach facilitates two key transitions necessary for achieving reduced carbon emissions in the long term: 1) it enhances the integration of renewable energy sources and 2) it increases the overall system efficiency by exploiting the interaction of different energy vectors. Figure 1 displays the single line diagram of the representative system used and highlights the interface of both thermal and electrical networks at the chiller plant level. The absorption chiller, which utilizes the heating power from the exhaust gases and jacket water loop of a gas engine (CCHP) to provide cooling power, is an example of one such unit that couples different energy vectors. The energy management strategy is in the form of a cloud-based application that performs a model predictive control (MPC) of the energy components and is described in [23]. At first, forecasts are performed for the electrical $\left(P_{t}^{l}\right)$ and thermal load profiles $\left(Q_{t}^{l}\right)$ along with the solar generation $P_{t}^{p v}$ defined 24 hours ahead (set $t \in T$ ). The objective of the EMS is then to minimize the energy bill (i.e. cost of electricity and gas) along the prediction horizon. This is performed while optimizing the schedules for the controllable energy assets that are the degrees of freedoms of the system - typically the battery and thermal storage charges $\left(P_{t}^{b a t+}\right.$, $\left.Q_{t}^{t s^{+}}\right)$and discharges $\left(P_{t}^{b a t-}, Q_{t}^{t s-}\right)$ as well as the gas engine generation $\left(P_{t}^{g e}\right)$. Note that the grid power over the prediction period is estimated in the course of the optimization with the input forecast and the values for the degree of freedom. The grid import and export flows $\left(P_{t}^{g d+}, P_{t}^{g d-}\right)$ finally allow to compute the electricity bill that is minimized. The chiller electrical consumption $\left(P_{t}^{c h}\right)$ and thermal generation $\left(Q_{t}^{c h}\right)$ are predicted in the same way along the optimization process. Note that the cooling power extracted from the absorption chiller $\left(Q_{t}^{a b c h}\right)$ is not directly controllable and depends on the gas engine generation with an additional electrical consumption for backing 
the absorption chiller auxiliaries such as the cooling water pumps $\left(P_{t}^{a b c h}\right)$. The EMS operates on a rolling window basis of 30 minutes intervals with updated forecast.

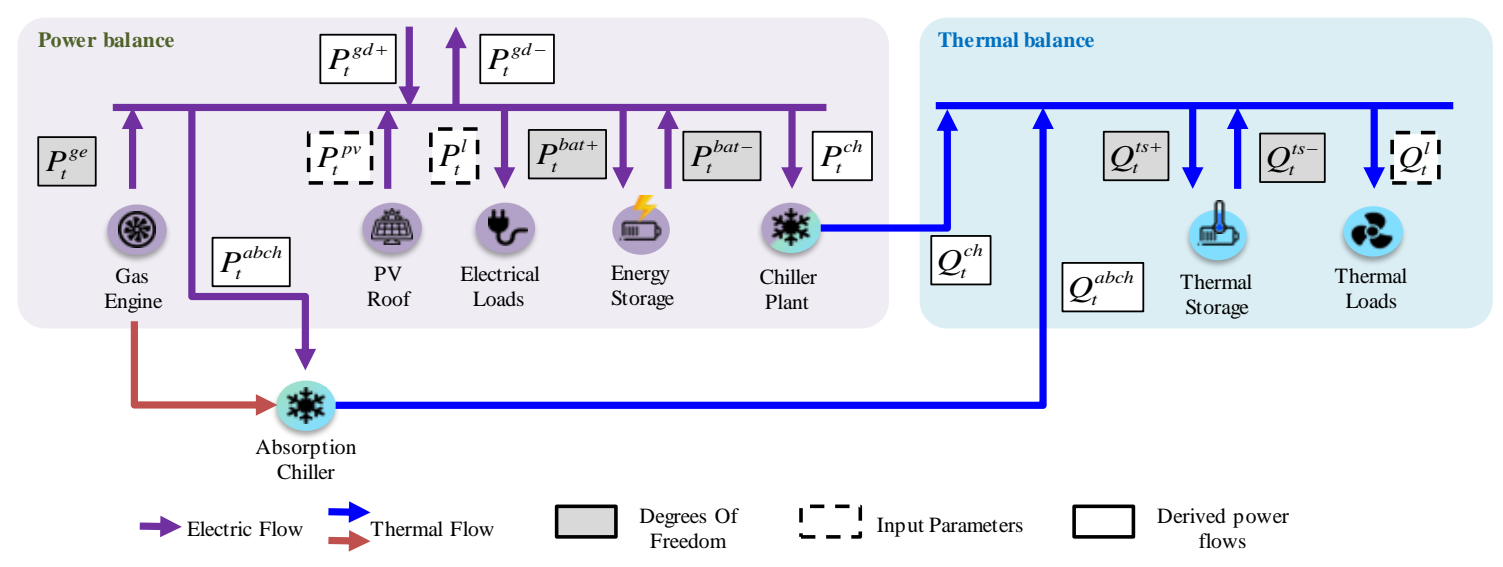

Figure 1: Considered multi-energy microgrid with both electrical/thermal buses and local load/generation

From the optimal planning perspective, the management strategy shall be simulated over a representative period and with different sets for the sizes of the energy components in order to find the best configurations. In this paper the simulation period considers a single day with reprehensive profiles for the solar radiation, and both thermal/electrical loads with maximum values that correspond to the peaks recorded over a year for the studied system. One day is obviously not representative enough to simulate the system operation over the expected lifetime. However, the main objective of the paper is the comparison of different optimization architectures for that type of planning problems. Considering longer times horizons increases the size of time set $T$ without changing intrinsically the nature of the problem. That point will be specifically addressed in Section 4.3.3 with additional test performed along weekly profiles.

The operating expenses of the multi-energy microgrid are computed with the EMS run only once in every case with the daily power profiles and with daily real-time electricity prices at $30 \mathrm{~min}$ resolution. Note that the photovoltaic (PV) generation varies with the size of the rated capacity of the generator $\left(P_{R}^{p v}\right)$. In practice, a normalized solar radiation profile is adopted and it is scaled depending on the PV generator capacity. The electricity rates $\pi_{t}^{e}$ are derived from the real time prices in Singapore while the gas correspond to a fixed tariff $\pi_{t}^{g}$ at $0.6 \$ / \mathrm{kg}$. The price has been set according to the HSFO trading price, with proper correction factors that take distribution and type of contract (i.e. take or pay) into consideration. It is important to note that a single day is not representative enough to emulate the operation of the system along its expected lifespan (typically decades). This is especially true for cases where uncertain inputs such as intermittent renewable generation, varying consumption patterns or fluctuating energy prices are considered. However, as the scope of the paper is mainly concerned with the interaction and integration of the management strategy into an optimal planning tool with different sizing frameworks, the representative profile of a single day is deemed sufficient.

\subsection{Optimal Power and Cooling Dispatch}

Previous works focused on the implementation of the optimal management problem have been solved with a Sequential Quadratic Programming (SQP) method embedded in MATLAB [24]. As mentioned previously, the simulation estimates the operating cost in the framework of the optimal planning of the system. This section describes the mathematical formulation of the problem. The objective function that is minimized is the total energy bill that considers the electricity prices $\pi_{t}^{e}$ for the power imported from the grid 
$\left(P_{t}^{g d+}\right)$ and gas price $\pi_{t}^{g}$ associated with the fuel consumption of the gas engine $M_{t}^{g e}$ per unit time. These prices are defined as time series parameters along the simulated day with a time step $\Delta t=30 \mathrm{~min}$ (1). Note that the system representation considered here lies in steady state modeling. The power/cooling generated or consumed by the different assets is supposed to be constant between two time intervals. No differential equations are considered and the model remains at the powers level (i.e. voltage/current not represented on the electrical side, pressure/flow not represented on the thermal side). That approach is systematically used in planning problem or optimized operations over long periods of time (day, months, and years). Transient models and finer time representation would led to an increase problem complexity and unsolvable problems.

$$
\text { obj }: \min \sum_{t=1}^{T}\left(P_{t}^{g d+} \times \pi_{t}^{e}+M_{t}^{g e} \times \pi_{t}^{g}\right) \times \Delta t
$$

Different sets of constraints are then introduced to represent the operating conditions of the different components. The equations for the gas engine and chiller plant operations refer to quadratic models. The different efficiencies and state variables depend on the rated capacities of the assets, $P_{R}^{g e}$ for the gas engine and $Q_{R}^{c h}$ for the chiller. Those "reference" models for the components can be interpreted as functions $P_{t}^{g e}=\mathrm{f}\left(M_{t}^{g e}, P_{R}^{g e}\right)$ and $P_{t}^{c h}=\mathrm{g}\left(Q_{t}^{c h}, Q_{R}^{c h}\right)$ using a normalized map approach [25] - with the gas engine output power $P_{t}^{g e}$ (in $\mathrm{kW}$ ) computed with the gas mass flow rate $M_{t}^{g e}$ (in $\mathrm{kg} / \mathrm{s}$ ) and the chiller electrical consumption $P_{t}^{c h}$ (in $\mathrm{kW}$ ) computed with the cooling generation $Q_{t}^{c h}$ (in RT). Note that the models specifically compute the efficiency of the assets on their whole operating range and with regards to the nominal value at the design conditions.

Different set of constraints are then defined for all the other components. Those operating constraints are in the form of linear equalities or inequalities. At first, for the energy storages units (both electrical and thermal), the charge/discharge powers $\left(P_{t}^{b a t{ }^{+}}, P_{t}^{b a t-}\right.$ and $\left.Q_{t}^{t s+}, Q_{t}^{t s-}\right)$ should remain within the rated values $\left(P_{R}^{b a t}\right.$ and $\left.Q_{R}^{t s}\right)(2)$. Typical formulation are considered with the storage efficiencies $\left(\eta^{b a t}, \eta^{t s}\right)$ when computing the state of charge at each time step depending on their initial values $\left(S O C_{0}^{b a t}, S O C_{0}^{t s}\right)$ and rated capacities $\left(E_{R}^{b a t}, E_{R}^{t s}\right)(3)$. Constraint (4) ensures that the states of charge remains within the specified limits and that the storage units arrive back to their initial SOC at the end of the simulated day (with |.| denoting the cardinality function). Note that, typically an inequality constraint is enough to impose the final value for the state of charge [18]. Indeed, with the minimization of the energy bill, any remaining energy stored at the end of the day can be interpreted as a 'lost' benefit as deeper discharge could avoid the use of other resources (e.g. grid or gas engine) attached with energy prices.

$$
\begin{aligned}
& \left\{\begin{array} { l } 
{ 0 \leq P _ { t } ^ { \text { bat+ } } \leq P _ { R } ^ { \text { bat } } } \\
{ 0 \leq P _ { t } ^ { \text { bat- } } \leq P _ { R } ^ { \text { bat } } }
\end{array} \text { and } \quad \left\{\begin{array}{l}
0 \leq Q_{t}^{t s+} \leq Q_{R}^{t s} \\
0 \leq Q_{t}^{t s-} \leq Q_{R}^{t s}
\end{array}\right.\right. \\
& \left\{\begin{array}{l}
S O C_{t}^{b a t}=S O C_{0}^{b a t}-\sum_{i=1}^{t}\left(\frac{P_{i}^{b a t-}}{\eta^{b a t}}-\eta^{\text {bat }} \times P_{i}^{\text {bat }}\right) \times \frac{100 \times \Delta t}{E_{R}^{b a t}} \\
S O C_{t}^{t s}=S O C_{0}^{t s}-\sum_{i=1}^{t}\left(\frac{Q_{i}^{t s-}}{\eta^{t s}}-\eta^{t s} \times Q_{i}^{t s+}\right) \times \frac{100 \times \Delta t}{Q_{R}^{t s}}
\end{array}\right.
\end{aligned}
$$

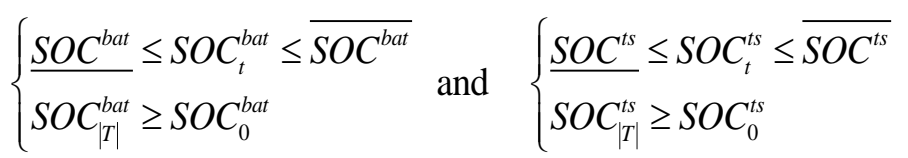


Equation (5) computes the cooling power $Q_{t}^{a b c h}$ generated by the absorption chiller with the coefficient of performance (COP $\left.{ }^{a b c h}\right)$ and electric power over waste heat ratio $\left(\beta_{p w h}^{a b c h}\right)-3.517$ as the conversion ratio between RT and kW. Note that the cooling power generation from the absorption chiller is also associated to the electrical consumption of the pumps that is computed with a fixed cooling to power coefficient $\beta_{p c r}^{a b c h}$. Furthermore, the power imported/exported from/to the main grid has to remain within the limit that can be interpreted as the contracted capacity of the considered site (6). The maximum value is set to $1600 \mathrm{~kW}$, slightly higher that the maximum power purchased from the grid in a case where no local energy components is installed in the microgrid (i.e. null sizes for PV, storages and gas engine). In such a scenario, all the cooling power is generated by the conventional chiller plant while the grid supplies the total electrical demand.

$$
\begin{gathered}
\left\{\begin{array}{l}
Q_{t}^{a b c h}=\frac{C O P^{a b c h} \times \beta_{p w h}^{a b c h} \times P_{t}^{g e}}{3.517} \\
P_{t}^{a b c h}=\beta_{p c r}^{a b c h} \times 3.517 \times Q_{t}^{a b c h}
\end{array}\right. \\
\left\{\begin{array}{l}
0 \leq P_{t}^{g d+} \leq \overline{P^{g d}} \\
0 \leq P_{t}^{g d-} \leq P^{g d}
\end{array}\right.
\end{gathered}
$$

Finally, the fulfillment of the fundamental constraints (7) ensures the balance between supply and demand at both electrical and thermal buses with the generation stacked on the left side of the equations and the loads on the right side. Note that, with such a formulation, the electrical grid is often denoted as an 'infinite storage' where the limit $\overline{P^{g d}}$ is high enough at it is the case here. The idea is that every local imbalance is supplied by the grid within specific power bounds. However, the simulations were performed while setting the injection to the grid to $0((6))$. Indeed, for the considered system, no feed in tariff policy is considered for any excess of local generation exported to the main grid for additional benefit. The main reason is to encourage the self-consumption rather than installing oversized assets with regards to the local load. Thus, the convergence is guaranteed on the electrical side for every size for the energy components. However, on the thermal side, the balance constraints might not be fulfilled if the rated capacity of the chiller plant $Q_{R}^{c h}$ is not enough to supply the load considering the sizes of the absorption chiller and thermal storage. Thus, as it will be explained in the following sections, some microgrid configuration in terms of rated capacities might lead to a non-convergence of the management loop. In such a case, the operating cost will be associated to an infinite value in order to penalize the corresponding set of component sizes. Table 1 introduced a list of the used symbols and acronyms.

$$
\left\{\begin{array}{l}
P_{t}^{g d+}+P_{t}^{p v}+P_{t}^{g e}+P_{t}^{b a t-}=P_{t}^{l}+P_{t}^{c h}+P_{t}^{a b c h}+P_{t}^{b a t+}+P_{t}^{g d-} \\
Q_{t}^{c h}+Q_{t}^{a b c h}+Q_{t}^{t s-}=Q_{t}^{l}+Q_{t}^{t s+}
\end{array}\right.
$$

\section{COMPutational Time Reduction for Operating Cost Estimation}

\subsection{Generic Piecewise Linearization for the Component Models}

The 'reference' problem for the $O P E X$ estimation is originally solved using Sequential Quadratic Programing (SQP) with the MATLAB fmincon function. The observed computational time of around 5 min cannot comply with an optimal planning strategy that would require to estimates the $O P E X$ for a great number of different microgrid configuration $\left(10^{3}-10^{4}\right)$. Therefore Section 3 describes 
the time reduction of the operation loop with the use of a linearized formulation for the optimal scheduling problem (i.e. optimization over the representative day). That type of mathematical representation has been commonly used for more than a decade for optimal power dispatch in electrical microgrids [26] and for unit commitment problems that involve greater numbers of generation assets in transmission networks [27]. Such an approach requires linear models for the state variables attached to the different generating components. As previously mentioned, the nonlinearities of the considered system are related to the intrinsic physical behavior of the gas engine and vapor compression chiller that are typically summarized by polynomial expression of the second order. The performances depends on the sizes of the assets and a great number of different capacities is investigated in the framework of optimal planning.

Table 2: Generic piecewise linearization process based on least square minimization and applied to gas engine and chiller models

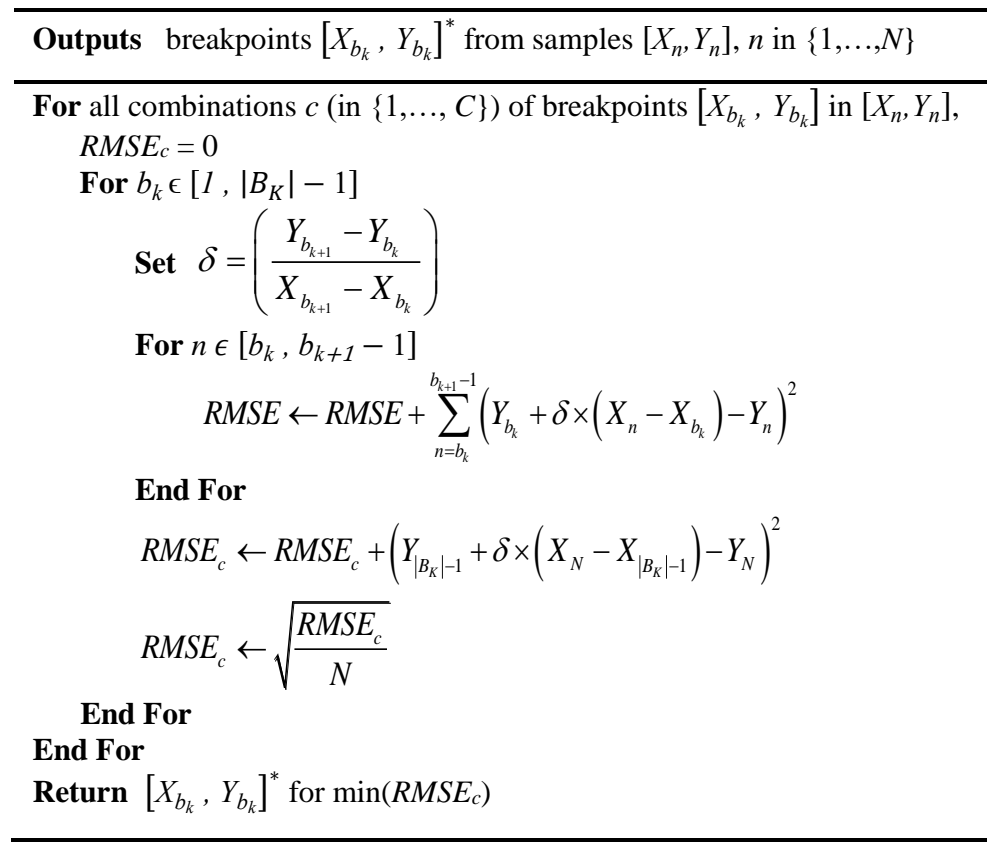

Each time a new system configuration is tested, the models for the gas engine and chiller allow both units to be simulated along their entire operating range for the given rated sizes. Thus, operating profiles are obtained with $N$ samples $\left[M_{n}^{g e}, P_{n}^{g e}\right]$ and $\left[Q_{n}^{c h}, P_{n}^{c h}\right]$ and a generic piecewise linearization method (PWL) is developed in order to determine in every case the set of breakpoints $B_{k}(\mathrm{i} . \mathrm{e}$. piecewise linear segments) that minimizes the root mean square error $\left(R M S E^{*}\right)$ with the reference model. Table 2 describes the implemented procedure that is based on the exhaustive search while considering all the possible combinations of breakpoints $B_{k}$ among the $N$ samples. The computation of the overall error involves the calculation of the RMSE in each block (i.e. between the breakpoints) with $\delta$ the slope of the corresponding piecewise linear segment. Figure 2 displays the results obtained when running the generic linearization for different sizes of the components. Very low deviations from the "reference" models are observed (less than $0.5 \%$ error) with only three piecewise blocks. 

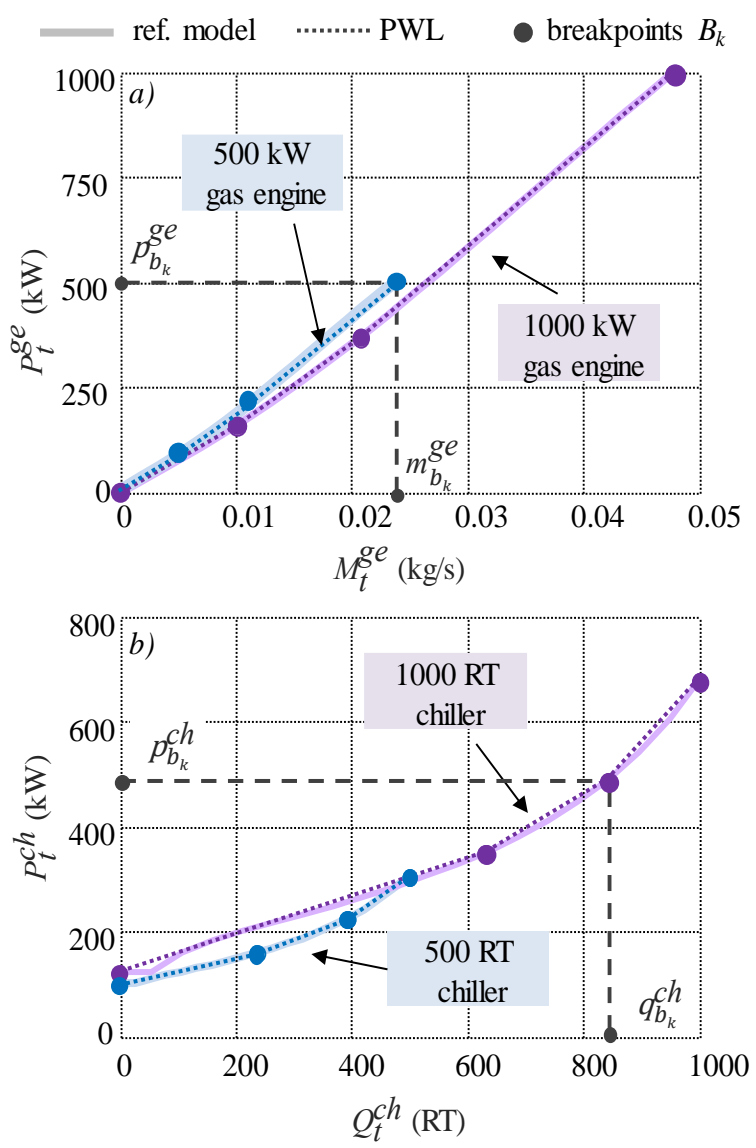

Figure 2: Comparison between reference quadratic models and piecewise linearization results ( 3 block) - a) different sizes of gas engines b) different sizes of chillers

\subsection{Mathematical Formulation}

Ultimately, the procedure described above returns the sets of optimal breakpoints for the gas engine $\left[m_{b_{k}}^{g e}, p_{b_{k}}^{g e}\right]$ and for the chiller plant $\left[q_{b_{k}}^{c h}, p_{b_{k}}^{c h}\right]$ that will be used in the optimal management strategy. Traditional PWL approaches in unit commitment problem consist in adding a set of continuous variables to approximate a convex function (typically quadratic generation cost) that is directly integrated in the objective function to minimize [28]. However, in the optimal management considered here, the linearized functions are not directly minimized and are integrated in the operating constraints (power balance equalities). Thus, a generic mathematical formulation involving both continuous and binary variables [29] is favored in order to implement the simplified models in the optimization for the power dispatch. Especially, weight coefficients $[0,1]$ are introduced, $\alpha_{b_{k}, t}^{g e}$ for the gas engine and $\alpha_{b_{k}, t}^{c h}$ for the chiller, while the PWL block $k$ in which the components operate are identified with binary variables - $u_{k, t}^{g e}$ for the gas engine and $u_{k, t}^{c h}$ for the chiller. A set of constraints should then be fulfilled in order to represent the equipment operating conditions. Constraint (8) ensures that only one operating block is identified if the gas engine is running (i.e. $u_{t}^{g e}=1$ ) while (9) imposes non null weights around the operating segment only and the summation of the non null weight should be equals to 1 (10). Finally, the operating conditions in terms of mass flow rate and output power for the gas engine are computed as a weight sum of the different PWL breakpoints previously defined (11) and the operating powers shall remain within the rated values (12). Figure 3 displays an example for a gas engine and a two blocks linearization 
with the equipment operating between the second and third breakpoints. Note that similar sets of variables, parameters and constraints are introduced in order to represent the running state of the vaporized chiller plant.

$$
\begin{aligned}
& \sum_{k=1}^{|K|} u_{k, \mathrm{t}}^{g e}=u_{t}^{g e} \\
& \left\{\begin{array}{l}
\alpha_{b_{k}, t}^{g e} \leq u_{k, t}^{g e} \text { if } b_{k}=1 \text { and } k=1 \\
\alpha_{b_{k}, t}^{g e} \leq u_{k, t}^{g e} \text { if } b_{k}=B_{K} \text { and } k=K \\
\alpha_{b_{k}, t}^{g e} \leq u_{k-1, t}^{g e}+u_{k, t}^{g e} \text { otherwise }
\end{array}\right. \\
& \sum_{b_{k}=1}^{\left|B_{K}\right|} \alpha_{b_{k}, t}^{g e}=u_{t}^{g e} \\
& M_{t}^{g e}=\sum_{b_{k}=1}^{\left|B_{K}\right|} \alpha_{b_{k}, t}^{g e} \times m_{b_{k}}^{g e} \quad \text { and } P_{t}^{g e}=\sum_{b_{k}=1}^{B_{K} \mid} \alpha_{b_{k}, t}^{g e} \times p_{b_{k}}^{g e}
\end{aligned}
$$

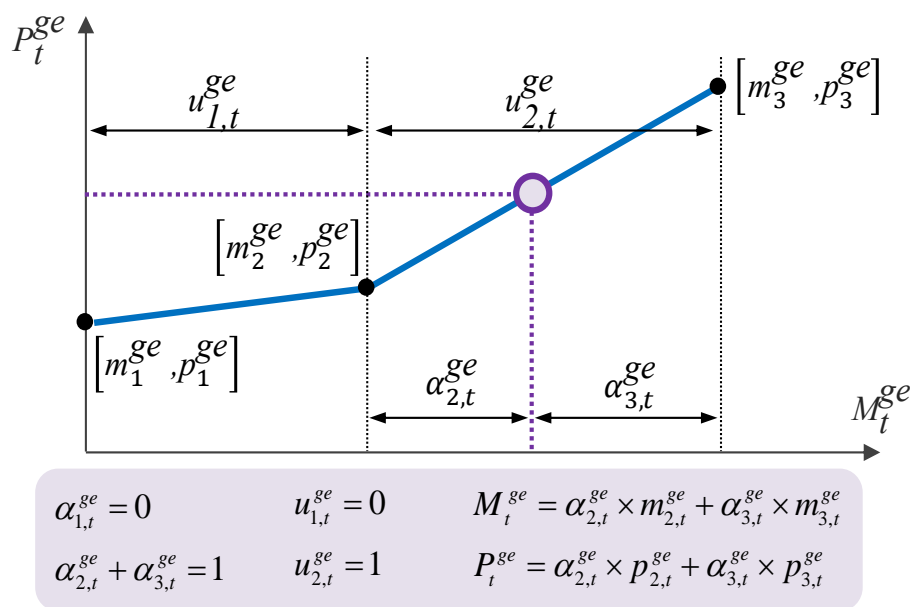

Figure 3: Mixed Integer Linear Programming formulation for the gas engine operation using the piecewise linearization outputs

\subsection{Validations Runs and Obtained Results}

As already mentioned, Section 3 focuses on time reduction of the dispatch problem while moving from SQP to Mixed Integer Linear Programming (MILP). This is done thanks to the piecewise linearization process described in Section 3.1 and using the mathematical modeling in Section 3.2. Section 3.3 allows to validate the approach with the comparison of the results (in terms of operating costs) obtained with the linearized models and the reference equation of Section 2.2. The MILP optimal dispatch problem is formulated in MATLAB using YALMIP [30] and solved with CPLEX 12.8.1 (16 threads in parallel, 32 GB RAM, 2.7GHz processor). A first set of simulations is performed with arbitrary values for the different energy components $-250 \mathrm{kWp}$ for the solar generator, a $300 \mathrm{~kW} / 300 \mathrm{kWh}$ battery, a $50 \mathrm{RT} / 300 \mathrm{RTh}$ thermal storage and a $1000 \mathrm{~kW}$ gas engine. Note that the assumption is made that the absorption chiller is sized according to the gas engine. The corresponding pump consumption and thermal generation are computed 
with the gas engine output power (5) and are not subject to any other constraint. Table 3 compares the results obtained when running the optimal management with different number of PWL blocks and with regards to the reference results return with the original quadratic models. Logically, the computational time increases with higher values of $K$ that correspond to greater numbers of variables and constraints. However, the observed values are below one second. The $O P E X$ tends to decrease when more PWL segments are modeled, which suggest that the linearization underestimates the efficiency of the gas engine. Indeed, the results of the optimal dispatch with the "reference" model display an $O P E X$ value of 2421 \$, lower than the performances of the MILP formulation. Finally, the error between the approximate model and the reference case is very low, with a difference lower than $1 \%$ when more than three PWL blocks are considered while the computational time is reduced almost thousand times.

Table 3: Validation runs for the piecewise linearization process compared to the reference quadratic models for the computation of the system operating costs

\begin{tabular}{ccccccc}
\hline $\boldsymbol{K}$ & $\mathbf{2}$ & $\mathbf{4}$ & $\mathbf{6}$ & $\mathbf{8}$ & $\mathbf{1 0}$ & $\begin{array}{c}\text { Ref. } \\
\text { SQP }\end{array}$ \\
\hline OPEX (\$) & 2469 & 2441 & 2440 & 2436 & 2437 & 2421 \\
\hline CPU (s) & 0.32 & 0.35 & 0.51 & 0.52 & 0.69 & 300 \\
\hline Err. (\%) & 2.00 & 0.83 & 0.81 & 0.63 & 0.66 & - \\
\hline
\end{tabular}

A second set of simulations is then performed while estimating the $O P E X$ for twenty random configurations. Those configurations are randomly sorted using Latin Hypercube Sampling (LHS) in order to uniformly cover the search space along every dimensions (Table 4).

Table 4: Test system configurations (installed capacities) for validation runs of the pricewise linearization

\begin{tabular}{cccccc}
\hline Config. & $\begin{array}{c}\boldsymbol{P}_{\boldsymbol{R}}^{\boldsymbol{p}} \\
(\mathrm{kW})\end{array}$ & $\begin{array}{c}\boldsymbol{P}_{\boldsymbol{R}}^{\boldsymbol{g e}} \\
(\mathrm{kW})\end{array}$ & $\begin{array}{c}\boldsymbol{E}_{\boldsymbol{R}}^{\boldsymbol{a a t}} \\
(\mathrm{kWh})\end{array}$ & $\begin{array}{c}\boldsymbol{E}_{\boldsymbol{R}}^{\boldsymbol{s}} \\
(\mathrm{RTh})\end{array}$ & $\begin{array}{c}\boldsymbol{Q}_{\boldsymbol{R}}^{\boldsymbol{c h}} \\
(\mathrm{RT})\end{array}$ \\
\hline $\mathbf{1}$ & 0 & 1273 & 620 & 148 & 790 \\
\hline $\mathbf{2}$ & 404 & 1925 & 1177 & 490 & 971 \\
\hline $\mathbf{3}$ & 233 & 1310 & 1740 & 68 & 761 \\
\hline $\mathbf{4}$ & 332 & 427 & 1889 & 192 & 810 \\
\hline $\mathbf{5}$ & 134 & 0 & 1448 & 472 & 995 \\
\hline $\mathbf{6}$ & 262 & 1806 & 516 & 235 & 537 \\
\hline $\mathbf{7}$ & 367 & 1475 & 1208 & 168 & 550 \\
\hline $\mathbf{8}$ & 484 & 247 & 250 & 96 & 513 \\
\hline $\mathbf{9}$ & 197 & 105 & 172 & 450 & 883 \\
\hline $\mathbf{1 0}$ & 96 & 573 & 435 & 118 & 840 \\
\hline $\mathbf{1 1}$ & 50 & 1775 & 308 & 211 & 674 \\
\hline $\mathbf{1 2}$ & 276 & 1607 & 949 & 403 & 943 \\
\hline $\mathbf{1 3}$ & 376 & 993 & 0 & 274 & 855 \\
\hline $\mathbf{1 4}$ & 322 & 1070 & 1580 & 327 & 616 \\
\hline $\mathbf{1 5}$ & 163 & 341 & 1389 & 322 & 598 \\
\hline $\mathbf{1 6}$ & 434 & 1180 & 1970 & 354 & 687 \\
\hline $\mathbf{1 7}$ & 218 & 1536 & 760 & 0 & 916 \\
\hline $\mathbf{1 8}$ & 456 & 620 & 1058 & 277 & 632 \\
\hline $\mathbf{1 9}$ & 61 & 750 & 1669 & 47 & 712 \\
\hline $\mathbf{2 0}$ & 114 & 835 & 831 & 387 & 730 \\
\hline & & & & &
\end{tabular}

The $O P E X$ is computed in every cases with the 'reference' models and the linearized constraints. The results displayed in Figure 4 show small deviations and most importantly, two sets of model are capable of equally ranking the 'cheapest' or 'most expensive' configurations. Thus, the rank of the solutions (i.e. based on cost performances) is systematically respected when the optimal management is run with the different methods which implies that using faster linearized models in a planning procedure (i.e. to find the best configurations) would return the same results. As previously observed, the cost difference between the methods is very small (below 
$1 \%$ in most cases between PWL with $K=3$ and the "reference"), with a computational time of ten seconds maximum for the MILP and up to more than five minutes for the "reference" model. Lastly, note that two of the tested microgrid configurations do not converge regardless of the method, which is due to small sizes for the chiller as aforementioned.

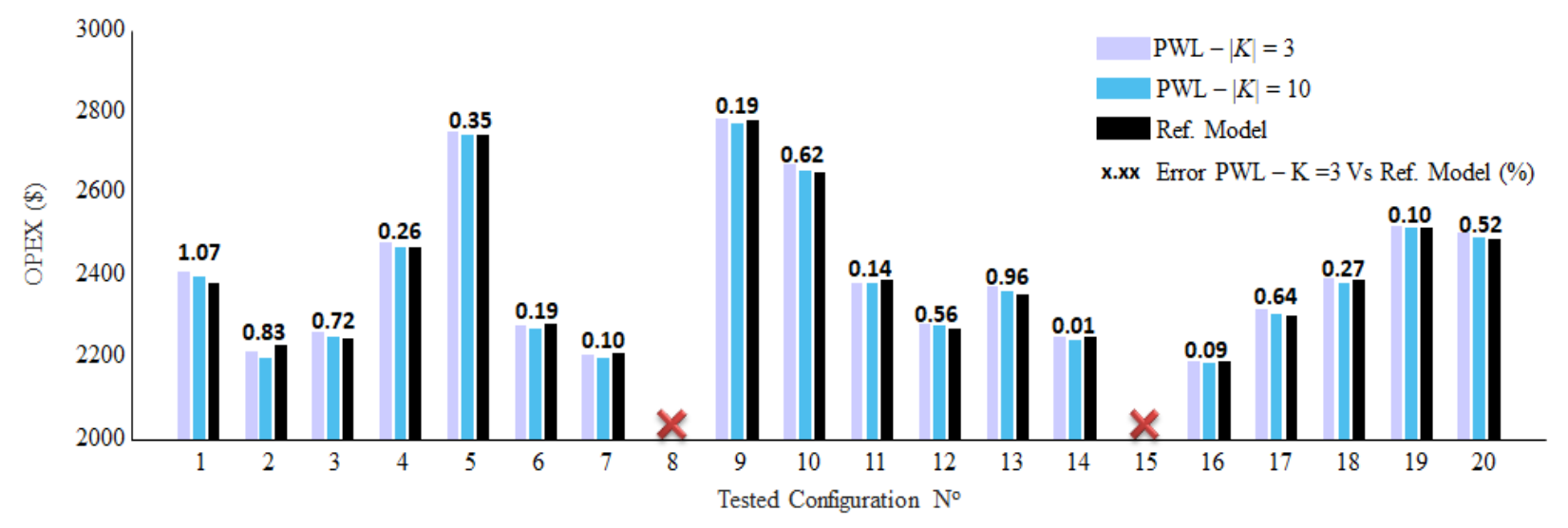

Figure 4: Operating cost estimation computed with the 'reference' models and linearized formulations for 20 different system configurations

\section{Frameworks for Optimal Planning}

\subsection{Optimal Planning Problem}

Section 3 focused on the computational time reduction of the operating cost (OPEX) estimation with given rated capacities of the installed assets (i.e. solar generator, gas engine, etc). The dispatch problem is summarized as in (13) where the $O P E X$ is estimated over a representative day while optimizing the different operating variables Xop in order to minimize the overall energy bill.

$$
\mathbf{X}_{\mathbf{O P}}^{*}=\arg \min \left(O P E X\left(\mathbf{X}_{\mathbf{O P}}\right)\right) \text { with } \mathbf{X}_{\mathbf{O P}}=\left[\begin{array}{c}
P_{t=0}^{g e}, \ldots, P_{t=T}^{g e} \\
P_{t=0}^{b a t+}, \ldots, P_{t=T}^{b a t+}, P_{t=0}^{b a t-}, \ldots, P_{t=T}^{b a t-} \\
P_{t=0}^{g d+}, \ldots, P_{t=T}^{g d+}, P_{t=0}^{g d-}, \ldots, P_{t=T}^{g d-} \\
Q_{t=0}^{c h}, \ldots, Q_{t=T}^{c h} \\
Q_{t=0}^{t s+}, \ldots, Q_{t=T}^{t s+}, Q_{t=0}^{t s-}, \ldots, Q_{t=T}^{t s-}
\end{array}\right] \text { subject to constraints (2)-(12) }
$$

With the objective of optimal long term planning for the considered system described in this section, the operating cost has to be estimated along the capital expenditures $(C A P E X)$. That $C A P E X$ depends on the cost of the installed equipment with the rated capacities arranged in a 'sizing' vector $\mathbf{X}_{\mathbf{C A P}}=\left[P_{R}^{p v}, P_{R}^{g e}, E_{R}^{b a t}, E_{R}^{t s}, Q_{R}^{c h}\right]$. The sizes of the different assets have an impact on the $O P E X$ with the operating constraints introduced ((2)-(12)). Especially, every times different rated capacities are investigated for the gas engine and chiller, the PWL process defined previously is run in order to define the operation limits ((2)-(12)) with the appropriate breakpoints $(|K|=3)$. Therefore, both $O P E X$ and $C A P E X$ have to be 'co-optimized' in order to reach the best tradeoff. Indeed, if higher rated capacities (e.g. for the PV and battery) would give the opportunities for lower operating costs, those savings might not be significant enough to recover the initial investment at the end of the system expected lifespan. Traditional approaches consist in maximizing the Net Present Value $(N P V)$, which is defined as the sum of all the cash flow over the system duration ( $N_{y}$ equals to 20 years here) minus 
the initial investment costs with a discount rate $r$ and an utilization factor $\left(u_{f}=0.95\right)(14)$. However, for the considered system there is no cash flow in term of income. Thus, it is replaced in the NPV computation by the savings in the operating cost compared to a reference case and denoted $O P E X^{r e f}$. That base case is defined by the studied system with no solar, no CCHP, no storages and a 700 RT chiller plant. Preliminary tests showed that the $N P V$ values after twenty years varies a lot with only small deviations of the computed $O P E X-$ with only a $2 \%$ difference in $O P E X$ the $N P V$ value doubles. It is necessary to note that the operating cost estimation is only done over a single day and is subject to uncertainties for the renewables generation, user consumption and prices profiles. Implementing an optimal planning procedure with the maximization of the $N P V$ as the objective function will thus not be robust enough in terms of obtained solutions. As such, the annualized TCO of the system is preferred here as an objective for the planning problem with less sensitivity to the OPEX deviations (15). The optimal design then consists in minimizing the TCO with the operating cost computed over the representative day.

$$
\begin{aligned}
& \text { obj: } \max N P V\left(\mathbf{X}_{\mathrm{CAP}}\right)=\frac{r \times\left(1-r^{N_{y}}\right)}{1-r} \times\left(\text { OPEX } X^{r e f}-\operatorname{OPEX}\left(\mathbf{X}_{\mathrm{OP}}^{*}\right)\right) \times u_{f}-\operatorname{CAPEX}\left(\mathbf{X}_{\mathrm{CAP}}\right) \\
& \text { obj: } \min T C O\left(\mathbf{X}_{\mathrm{CAP}}\right)=\operatorname{OPEX}\left(\mathbf{X}_{\mathrm{OP}}^{*}\right) \times u_{f} \times 365+\frac{\operatorname{CAPEX}\left(\mathbf{X}_{\mathrm{CAP}}\right)}{N_{y}}
\end{aligned}
$$

Ultimately, the optimal planning consists in computing the best $T C O$ (or NPV) while determining the most appropriate sizes for the installed assets. Note that the OPEX is computed for every investigated configuration as depicted in (14) and (15). The bounds for the different equipment's ratings are set depending on the peak load level (1.5MW electrical and 800 RT cooling). Note that the maximum values for the gas engine (2 MW) and chiller (1000 RT) are significantly greater. The objective is to cover cases in which the optimal results correspond to a total load (with the storages charge) that may be higher than the baseline values.

- $P_{R}^{p v}$ varies within $[0 \mathrm{kWp}, 500 \mathrm{kWp}]$

- $P_{R}^{g e}$ varies within $[0 \mathrm{~kW}, 2000 \mathrm{~kW}]$

- $E_{R}^{\text {bat }}$ varies within $[0 \mathrm{kWh}, 2000 \mathrm{kWh}]$-with $P_{R}^{\text {bat }}$ varying in the same time within [0 kW, $\left.2000 \mathrm{~kW}\right]$

- $E_{R}^{t s}$ varies within [0 RTh, $500 \mathrm{RTh}$ - with $Q_{R}^{t s}$ remaining fixed at $50 \mathrm{RT}$

- $Q_{R}^{c h}$ varies within [500 RT, $\left.1000 \mathrm{RT}\right]$

The capital expenditures are computed with the different sizes of the components. Simple coefficients are considered with $750 \$ / \mathrm{kWp}$ for the solar system [32], $435 \$ / \mathrm{kW}$ for the absorption chiller (with regards to the gas engine rated power) [33]. The gas engine cost function depends on its rated power and efficiency (which is itself computed with the nominal output) and is mapped from the data advised in [33]. The cost for the battery is set at $795 \$ / \mathrm{kWh}$ including the modules replacement based on the system expected lifespan [34].

\subsection{Investigated Optimization Architectures}

\subsubsection{Iterative \& Integrated Approaches}

Conventional methods for planning studies consist in bi-level frameworks where an inner loop runs the management strategy and is integrated in an outer loop that explores different designs. Typically approaches require many runs of the management procedures which motivated the time reduction presented in the previous section with the use of MILP formulation. As depicted in Figure $5 a$, for 
every investigated design, the piecewise linearization process and the power dispatch problem are runs in order to estimate the operating cost, necessary to compute the TCO. The challenge in such bi-level approaches is to define the best way to generate the different capacities $\mathbf{X}_{\mathbf{C A P}}$ that has to be investigated in order to find the best configuration. At first, a simple iterative approach can consist in a typical Monte Carlo simulation where different configurations are successively tested. In this paper Latin Hypercube Sampling (LHS) is considered in order to guarantee that the investigated points are uniformly distributed in the search space with a higher diversity in the tested configuration compared to other random samplings [35]. That iterative approach is denoted LHS+MILP (Figure 5b) and consists in 10,000 random samples (different $\mathbf{X}_{\mathbf{C A P}}$ ). Other approaches involve the use of optimization procedures to explore the search space defined by the equipment ratings with integrated bi-level optimization. In this paper, evolutionary algorithms are first considered with the use of a GA (function embedded in MATLAB) and a classical PSO [37]. Those methods consist in metaheuristic procedures that imitate mechanisms inspired by biological processes (e.g. firefly, ant colony, etc) to select and generate the family of solutions to investigate. Their parametrization allows a compromise to be achieved between the exploration of the search space and the exploitation of the 'interesting' area regarding the objective function (i.e. the TCO here). For the GA, 100 generations are considered with a population size of 10 individuals which is deemed sufficient to explore the search space (5 sizing variables here). Similarly, the PSO is parametrized with 10 particles and 100 iterations at the maximum. A Sequential Quadratic Programming (SQP) is also investigated for the outer sizing loop. The embedded fmincon function in MATLAB is run with 100 iterations and 10,000 evaluations of the objective functions (i.e. the TCO here) at the maximum. Note that for all the bi-level (integrated) approaches, the main concern is to control the maximum computational time for the optimal planning. One evaluation of the objective function is mainly impacted by the computation of the operating cost using MILP and requires between 0.3-0.6 s depending on the assets rated capacities. Thus the 10,000 limit allows to maintain the computation of the planning below a couple of hours (while considering the execution of the sizing loop). The integrated approaches are finally denoted GA+MILP, PSO+MILP and SQP+MILP and are summarized in Figure $5 b$.

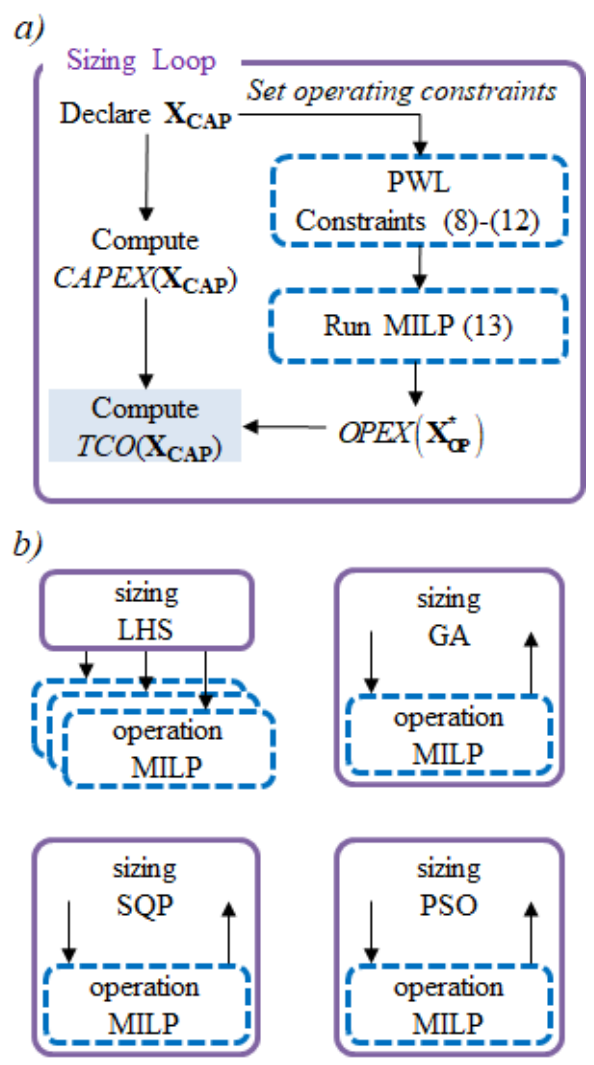


Figure 5: Optimization architectures to solve the planning problem - a) bi-level optimization architectures with MILP for the operating cost estimation - b) Investigated frameworks, iterative : LHS+MILP, integrated : GA+MILP, PSO+MILP, SQP+MILP

\subsection{2 'All-in-one’ SQP-Based Procedure}

An original 'all-in-one' procedure is implemented and consists in considering both sizing values (i.e. rated capacities) and controls (i.e. power flows) as variables of a single optimization problem. Similar approaches encountered in the literature usually consist in moving from a bi-level optimization to a single Mathematical Problem with Equilibrium Constraints (MPEC). It is achieved by representing the inner loop management by its equivalent KKT conditions when it can be proved that the corresponding problem is purely convex [19]. The method implemented here integrates the sizing variables to the original problem for the $O P E X$ solved with SQP (Section 2) and defines the TCO computation as the objective function to minimize.

$$
\mathbf{X}^{*}=\arg \min (T C O(\mathbf{X})) \text { with } \quad \mathbf{X}=\left[\begin{array}{ll}
\mathbf{X}_{\mathbf{O P}} & \mathbf{X}_{\mathbf{C A P}}
\end{array}\right]
$$

It is important to note that in the 'all-in-one' problem formulation, no binary variables are involved as the original quadratic constraints mentioned in section 2 are considered for the gas engine and chiller plant operations. Also, as detailed in Table 5 the total number of variables is obviously increased compared to the SQP problem that only considers the operating cost optimization. Also the operating bounds (e.g. (2)) become linear constraints between control and sizing variables.

Table 5: Comparison of SQP problem characteristic for the operating cost estimation with quadratic 'reference' models and the 'all-in-one' procedure for optimal planning

\begin{tabular}{ccc}
\hline & $\begin{array}{c}\text { Operation } \\
\text { SQP } \\
\text { (Section 2) }\end{array}$ & $\begin{array}{c}\text { 'all-in-one' } \\
\text { SQP }\end{array}$ \\
\hline Sizing Variables & 0 & 5 \\
\hline Operation Variables & 384 & 384 \\
\hline TOTAL Variables & $\mathbf{3 8 4}$ & $\mathbf{3 8 9}$ \\
\hline Bounds Constraints & 768 & 490 \\
\hline Linear Inequality Constraints & 242 & 530 \\
\hline Nonlinear Equality Constraints & 96 & 96 \\
TOTAL Constraints & $\mathbf{1 1 0 6}$ & $\mathbf{1 1 1 6}$ \\
\hline
\end{tabular}

\subsection{Results}

\subsubsection{Algorithms Performances}

Table 6 summarizes the results obtained after 10,000 random evaluations with LHS and ten runs of the others frameworks to solve the planning problem (average values with $95 \%$ confidence interval). Note that several runs are considered for the GA and PSO as they are stochastic methods that return different results even for a same starting population (or set of particle). On the contrary, SQP is a deterministic method and returns the same result every time it is run using the same starting point. However, that result may depends strongly on the starting point - starting point close to a local optimum, where the stopping criteria such as first and second order derivative and constraint tolerance are met. Multiple runs with different starting points are often adopted for more robustness [36]. Ten runs with distinct starting points are considered here. 
The best solution with the MILP formulation is obtained with the LHS at a cost of a time consuming search (more than 10 hours) and the PSO returns on average better solutions than the other integrated approaches. In addition, the confidence interval is small which denotes that the PSO tends to return consistent results no matter the initial position of the particle. On the contrary, the SQP results strongly depends on the initial starting point with a greater confidence interval after ten independent runs (non convergence for one run) even if the overall best solution displays a TCO close to the best PSO value. The integration of the faster MILP into bi-level optimization frameworks allows computational times from 23 min with the SQP to 1 h 30 min with the GA. As displayed in Figure 6, those values suggests that the different algorithms meets their stopping criteria before maximum number iterations/evaluation of objective functions (10,000 evaluations) in the case of bi-level (integrated) approaches. Such low computation times would have not been possible when considering an integrated design with the operating cost estimated with the "reference" quadratic models (i.e. around 5 min computation for the OPEX estimation with SQP compared to less than a second using MILP).

Table 6: Obtained Total Cost of Ownership (mean value and confidence interval) after 10 runs of the different investigated optimization architectures

\begin{tabular}{cccccc}
\hline & LHS+MILP & SQP+MILP & GA+MILP & PSO+MILP & All SQP \\
\hline Average $\boldsymbol{T C O}(\mathbf{k} \$ \mathbf{)}$ & N.A. & $936.7 \pm 29.2$ & $910.6 \pm 4.9$ & $891.4 \pm 2.3$ & $890.9 \pm 1.8$ \\
\hline Best $\boldsymbol{T C O}(\mathbf{k} \mathbf{)}$ & 888.9 & 890.2 & 897.2 & 890.1 & 887.7 \\
\hline Average CPU Time & N.A. & $23 \mathrm{~min}$ & $93 \mathrm{~min}$ & $89 \mathrm{~min}$ & $40 \mathrm{~s}$ \\
\hline
\end{tabular}

The most significant outcome from the different runs is that 'all-in-one' SQP approach displays outstanding performances with very low computational time below a minute and the convergence is reached at every run. Also the method is the most precise with the lowest confidence interval on the returned results after ten runs. Those high performances were unexpected considering that the approach embeds the nonlinear models for the gas engine and chiller plant. The running time of the 'all-in-one' problem is even shorter than the original SQP that only considers the computation of the operating cost while optimizing the management variables (around 5 min). At first glance, the overall design+operation would seem more complex with somewhat heterogeneous variables (rated capacity and variables power flow) and different classes of cost (i.e. CAPEX and OPEX). Nonetheless, as discussed in Section 4,2,2 and Table 5, the problem characteristics shows adding sizing parameters as optimization variables tends to increase the size of the feasible region compared to the overall search space. The number of variables is relatively more increased than the number of constraints - five more variables and ten constraints for the corresponding lower/upper bounds. Intuitively, the problem is simpler with more degrees of freedom to fulfill the constraints. For instance, the critical inequalities for power balances (7) may be reached while varying both operating and sizing variables. Also, considering the rated capacities as variables implies that the bounds for operating variables (2) and (12) become linear constraints between design and operation variables. With relatively more linear constraints the convergence is faster which is not uncommon for highly constrained problem solved using SQP methods as observed in [38]. Finally, it should be reminded that the comparison in terms of cost for the solutions from the 'all-in-one' SQP and those from the others approaches should be considered carefully. Indeed the models are not the same. Even if the previous subsection show very small deviations in terms of $O P E X$, the problems solved are not rigorously similar.

The final comment regarding the algorithms performances refers to the convergence speed of the evolutionary approaches. One of the main concerns when setting the parameters for the GA and PSO execution is to guarantee the exploration of the search space and not focusing on the interesting area only (unlike the 'all-in-one' SQP approach). The authors have presented a detailed paper on the methodology of setting up this kind of optimization problems (master-planning / optimal dispatch) in [39]. Figure 6 displays the 
convergence behavior after ten runs of the procedures. The GA tends to improve the objective quickly (in five generations only) but then tends to be "stuck" in the area close to the obtained solution. The optimal value returned (in terms of TCO) depends on the first stage of improvement and then differs a lot from one run to another which explains the greater confidence interval observed (Figure $6 a$ ). The PSO displays a slower convergence to better results. In most cases the procedure stops after 50 iterations due to the nonimprovement of the objective after 10 iterations (number of stall generations). As mentioned previously, the returned solution displays similar values with a narrow confidence interval (Figure 6b). Although the performances of the bi-level optimization framework with evolutionary algorithms are worse than the 'all-in-one' procedure, they are of interest when robust results are expected with algorithms that are capable of maintaining sufficient diversity of the returned solutions.
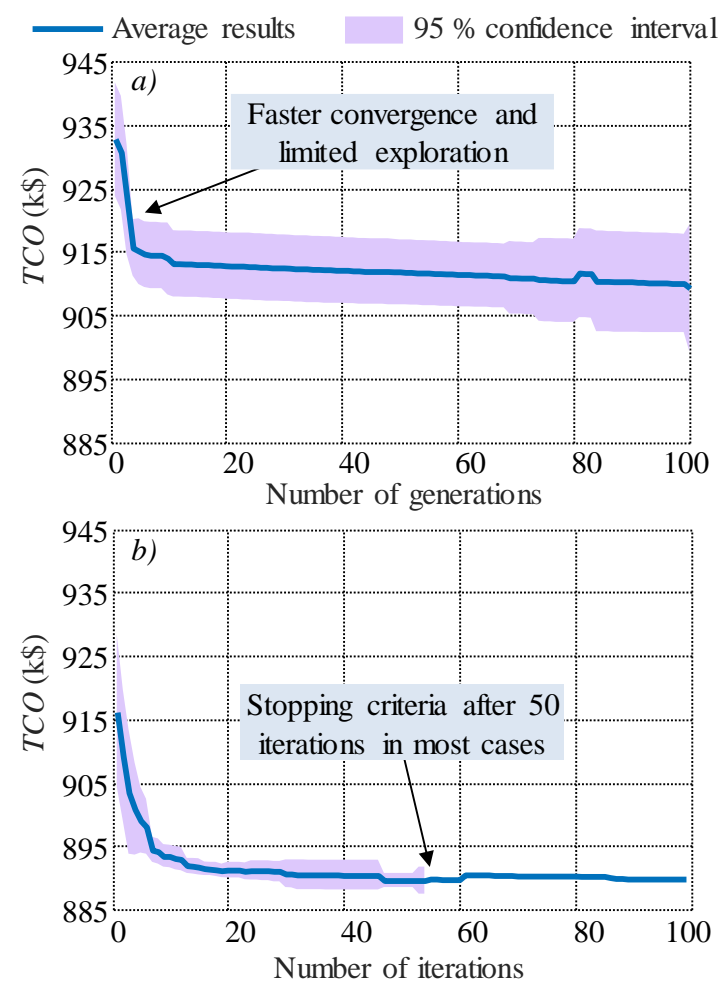

Figure 6: Evolutionary algorithms averaged performances after 10 runs - a) Genetic Algorithm - b) Particle Swarm Optimization

\subsubsection{Results Analysis}

The previous subsection focused on the algorithm performance in terms of the quality of the objective function solution and the computational time. In this subsection, the large number of solutions in the search space obtained from all the performed simulations, especially from the evolutionary algorithms and LHS runs, have been extracted and analyzed. Figure 7 displays 'solutions' that correspond not only to the optimal results returned by the procedures, but also to the set of investigated configuration in the course of the optimization. In each case the NPV, CAPEX and OPEX where computed a posteriori as the optimization only deals with TCO minimization. In order to avoid redundant points in the results analysis, close solutions with minimal difference in variables and TCO values were omitted from the solution space. This is especially true for the GA which may return a lot of solutions within the same neighborhood. 

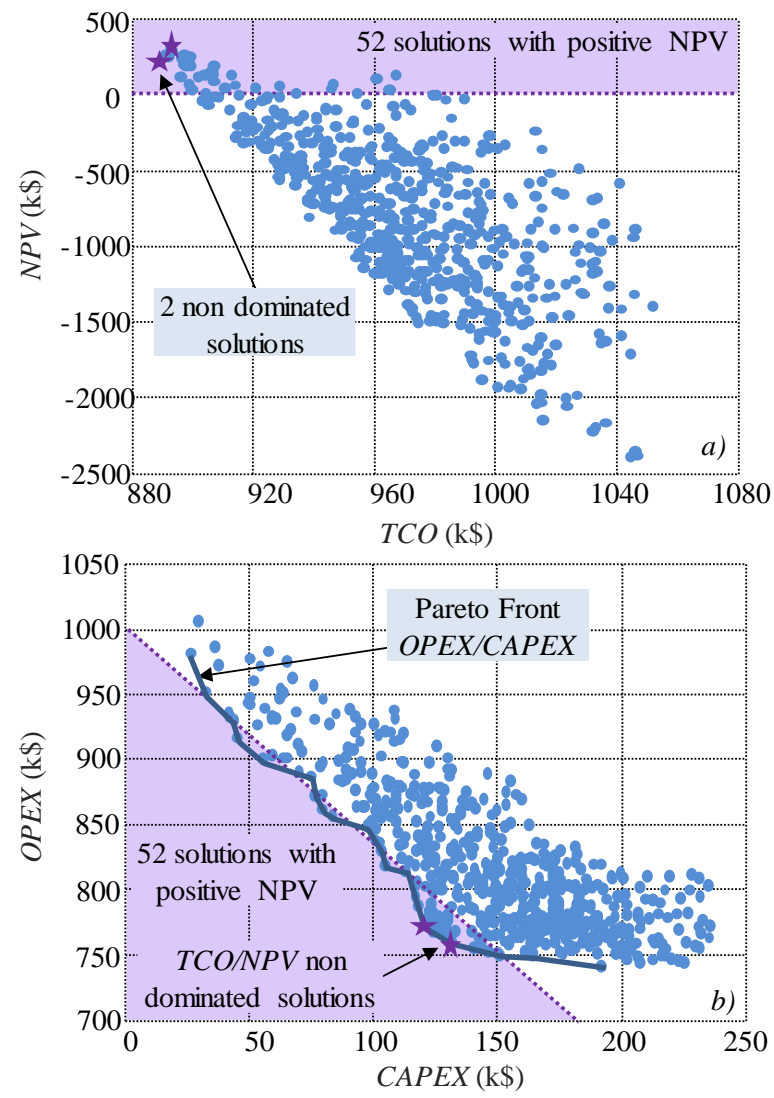

Figure 7: Result analysis with the pool of system configurations investigated using the different optimization architectures - a) Net Present Value Vs Total Cost of Ownership-b) Operating Costs Vs Capital Expenditures

The obtained results show that a few number of points correspond to a positive NPV at the end of the system lifetime (52 solutions out of 604) (Figure 7a). In other world, that implies that with the considered energy prices and cost for equipment the installed capacities, should be carefully considered to justify the investment (an optimal planning has to be performed). Those negative values are not wrong and are directly linked to the modeling of the economic environment. For instance, in the presence of low electricity rates with small absolute deviations, the installation of important storage capacities does not have practical sense. However, even if it is not discussed in the paper, other price policies, such as high penalty for the peak power, would result in increased optimal capacities. One noticeable outcome is that the optimizing the $T C O$ and $N P V$ are equivalent, in the optimal area only. The planning optimizes the TCO and the NPV is computed a posteriori in Figure $7 a$ with the best values corresponding to the same solutions, non-dominated in the Pareto sense. The choice was made to optimize the TCO as it is less 'noisy' regarding uncertainties/variations of the OPEX. As depicted on Figure7a, for the considered configuration the NPV varies in a ration from 1 to 6 while the TCO deviates from less than $20 \%$. Section $4,3,2$ has been modify in order to clarify those points.

Plotting the operating cost versus the capital expenditures highlights the challenge faced when solving systematic optimization problems (Figure 7b). As the distribution of points in Figure $7 b$ shows, larger capacities of the energy assets (especially storage and local generation) gives higher savings on the $O P E X$ but at the cost of higher initial investment. Thus, the optimization aims at obtaining the best compromise. The space of interesting solutions with positive $N P V$ values can be identified in the bottom part of the plotted area 
(i.e. lower $O P E X$ for a same $C A P E X$ ). Logically, the non-dominated solutions in terms of $T C O / N P V$ previously identified are located on the Pareto front of the $C A P E X / O P E X$ space.

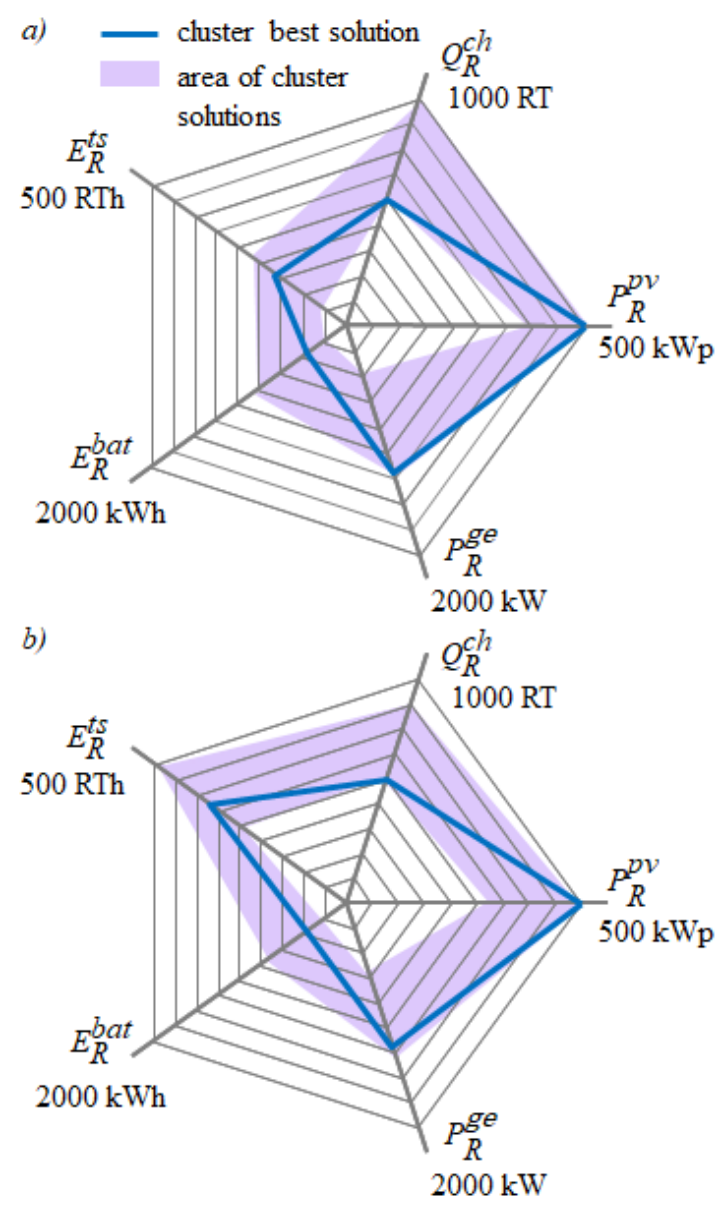

Figure 8: Partitioning of the obtained solutions with positive Net Present Value - a) Cluster 1-b) Cluster 2

A classical K-Means algorithm is then applied to the set of solutions with positive NPV in order to disaggregate the obtained points into two distinct clusters. The results displayed in Figure 8 show that the best solutions correspond to a high capacities for both the PV and gas engine. The main difference between the two groups of partitioned solutions lies in different arbitrages between the sizes of the storage components. Table 7 summarizes the solutions characteristics with the average values and confidence interval at $95 \%$ along with the values corresponding to the best non-dominated solutions in terms of TCO/NPV. The values for CAPEX and $O P E X$ are annualized with an utilization factor applied to the operating costs $\left(u_{f}=0.95\right.$ in $\left.(14)\right)$. Note that the difference between the specified range and the optimal value reflects that the best solution for each of the two clusters is not far from the cluster center. Such a clustering approach can be used in the framework of robust optimization where the objective would be to obtain areas with lower variations of the objective function (TCO here) when moving in the neighborhood of the optimal solutions. The confidence intervals observed are relatively small around the average solutions in terms of rated capacities and they display strong deviations of the $N P V$ (around $25-$ $30 \%$ ) that is more volatile than the TCO, CAPEX or OPEX. 
Table 7: Partitioning of the configurations investigated in the course of the optimization

\begin{tabular}{cccccccccc}
\hline & $\begin{array}{c}\boldsymbol{P}_{\boldsymbol{R}}^{\boldsymbol{v}} \\
(\mathrm{kW})\end{array}$ & $\begin{array}{c}\boldsymbol{P}_{\boldsymbol{R}}^{\boldsymbol{g e}} \\
(\mathrm{kW})\end{array}$ & $\begin{array}{c}\boldsymbol{E}_{\boldsymbol{R}}^{\text {bat }} \\
(\mathrm{kWh})\end{array}$ & $\begin{array}{c}\boldsymbol{E}_{\boldsymbol{R}}^{\boldsymbol{t s}} \\
(\mathrm{RTh})\end{array}$ & $\begin{array}{c}\boldsymbol{Q}_{\boldsymbol{R}}^{\boldsymbol{c h}} \\
(\mathrm{RT})\end{array}$ & $\begin{array}{c}T C O \\
(\mathrm{k} \$)\end{array}$ & $\begin{array}{c}\text { NPV } \\
(\mathrm{k} \$)\end{array}$ & $\begin{array}{c}\text { CAPEX } \\
(\mathrm{k} \$)\end{array}$ & $\begin{array}{c}\text { OPEX } \\
(\mathrm{k} \$)\end{array}$ \\
\hline Cluster 1 & $443 \pm 36$ & $913 \pm 192$ & $149 \pm 94$ & $102 \pm 31$ & $694 \pm 63$ & $920 \pm 13$ & $146 \pm 46$ & $106 \pm 15$ & $814 \pm 27$ \\
\hline Cluster 2 & $472 \pm 21$ & $981 \pm 146$ & $228 \pm 91$ & $365 \pm 32$ & $644 \pm 50$ & $911 \pm 9$ & $163 \pm 40$ & $116 \pm 11$ & $795 \pm 20$ \\
\hline Best Solution 1 & 500 & 1,207 & 198 & 142 & 500 & 888 & 309 & 130 & 759 \\
\hline Best Solution 2 & 500 & 1,163 & 111 & 36 & 525 & 890 & 355 & 123 \\
\hline
\end{tabular}

Finally, Figure 9 displays the operational results corresponding best solution of cluster 2 while plotting the stacked generation on both electrical and thermal sides. The daily profiles allow a better understanding of the planning outcomes. It can be observed that the local generation is maximized in order to avoid as much as possible any import from the grid that would incur greater $O P E X$. Thus, the PV capacity is set close to its upper bound and the gas engine reaches one MW rated so that the local assets supply the whole electrical demand when electricity prices are higher (i.e. with regards to the gas rates) (Figure $9 a$ ). On the plot depicting the thermal units, the absorption chiller provided a cheaper cooling power, extracted from the gas engine losses (Figure $9 b$ ). Note that when the storage charge durations can be identified every time the stacked generation exceed the demand curves. Figure $9 c$ allows to control that both electrical and thermal storages remain within the specified limits (10\%-90\% for the battery and 5\%-95\% for the thermal storage).
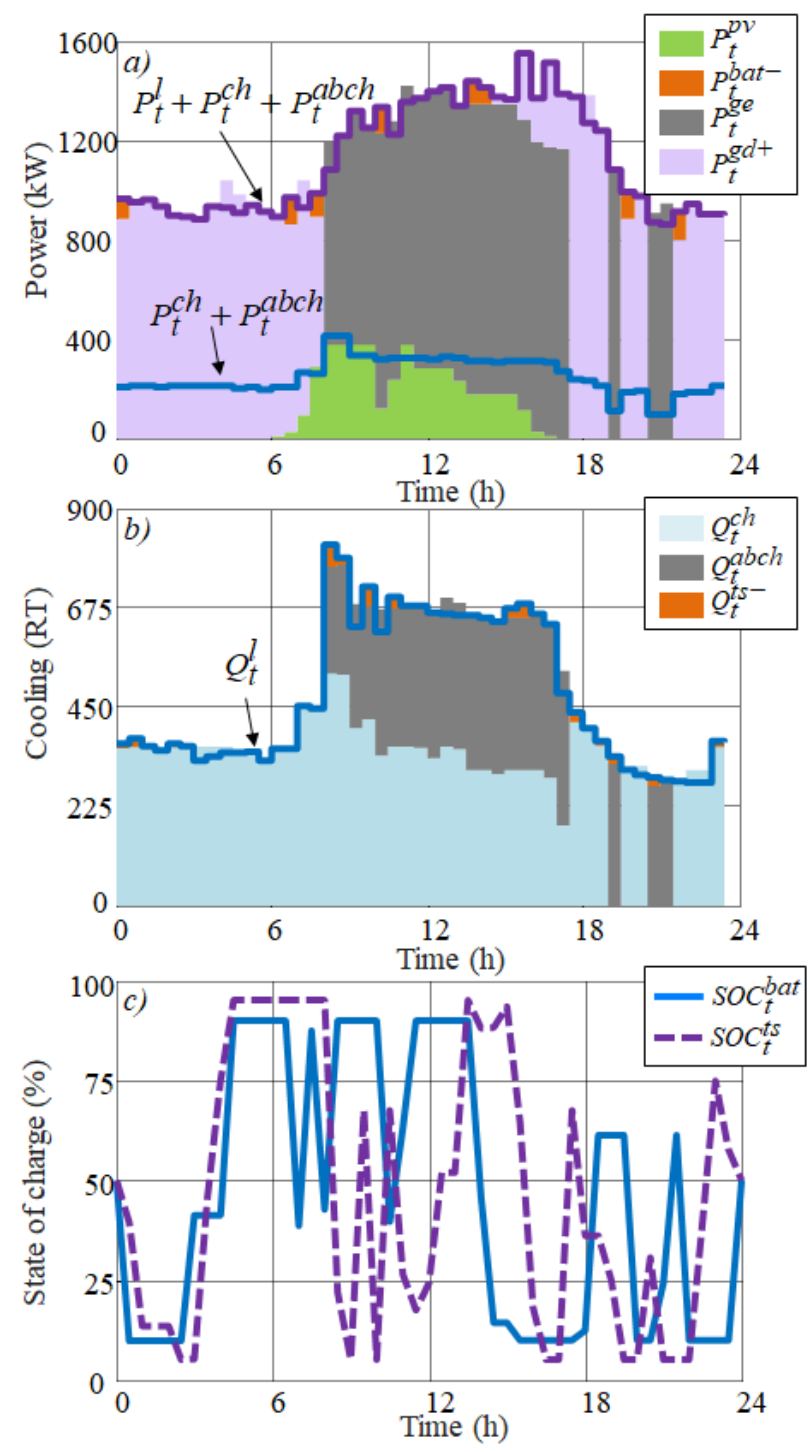
Figure 9: Power dispatch daily results for the optimal installed capacities corresponding to the best solution of cluster 2-a) Stacked electrical generation - b) Stacked cooling generation - c) State of charge

As already mentioned, the limitation imposed on the grid power avoids unrealistic sizes resulting from gaming with the energy prices by oversizing battery and gas engines. Note that the reduction of the peak power imported from the grid could also be considered in the objective function for the OPEX minimization. Indeed, with local generation and storage installed, the peak value may decrease at the same time as the amount of purchased electricity, which would result in additional savings. Thus, when such components are planned from the building design phase, the reduced size of the delivery transformer and the capacity subscribed to the power supplier can lower the investment costs. Further works should then consider those aspects along with other price policies and energy programs (e.g. demand response) that may encourage the installation of distributed resources. Finally, note that the gas engine is started up and shut down several times over the simulated day. The behavior is due to the absence of startup and shutdown costs that were omitted in this paper. Unlike unit commitment studies that refer to bigger power plants with larger power generators, the gas engine capacities considered here are in the range of $1 \mathrm{MW}$ and their start-up and shut down operation is flexible with minimal cost incurred.

\subsubsection{Longer Time Horizons}

This subsection discusses the representative period considered for the estimation of the operating costs of the system. Thus far, only a single day has been considered which is deemed insufficient to fully grasp the seasonality and variability of the different input parameters (e.g. solar radiation, load, energy prices, etc). However, is important to remind that the main objective of the paper is the investigation of different methodologies and optimization architectures. Especially, the performances of the different approaches are compared after many runs. Considering longer times profiles implies to extend the time set $T$ without affecting the nature of the problem (i.e. the objective/constraints remain the same over a longer time set). In addition, longer profiles increase the computational time and multiple runs of the different procedures, necessary for a performances comparison, may not be feasible in a reasonable duration. In spite of the aforementioned aspect, an additional set of optimization is performed with representative week that embed working and non working days for the office building considered in the study.

Table 8 displays the results obtained after 3 runs of the integrated and 'all-in-one' approaches. The LHS investigates 500 different configurations. Also, the stopping criteria of the different algorithms are scaled down in order to avoid prohibitive computational times (typically 30 iterations ate the maximum). The performances have to be considered with regards to Table 6 and similarities can be observed. Among the integrated architectures the PSO+MILP systematically returns the best results with small deviations good confidence while the SQP+MILP displays the greatest deviations. As for the daily simulations, the 'all-in-one' SQP is the most performant approaches in terms of obtained solutions. However, with weekly profiles, the time computation of the 'all-in-one' is in the range of the values observed for the other methods. That suggests the approach may not be scalable for longer simulated periods (40 s computation for one day and $3 \mathrm{~h}$ for one week). Finally, the obtained results in terms of optimal installed capacities are very consistent with the ones observed for a single day optimization (i.e. solar generator at the maximum bounds, gas engine around 1MW, small storage capacities and chiller plant big enough to fulfil the thermal balance constraints). Also, the results, and especially the best solutions, in terms of yearly TCO displays smaller values than the ones observed for the daily simulation. That is explained by the lower energy usage during the week-end resulting in smaller daily average. 
Table 8: Computational performances and best obtained result for the operating cost estimated over a representative week

\begin{tabular}{cccccc}
\hline & LHS+MILP & SQP+MILP & GA+MILP & PSO+MILP & All SQP \\
\hline Average $\boldsymbol{T C O}(\mathbf{k} \mathbf{)}$ & N.A & $906 \pm 25.4$ & $880 \pm 9.0$ & $846 \pm 1.1$ & $838 \pm 0.4$ \\
\hline Best $\boldsymbol{T C O}(\mathbf{k} \mathbf{)}$ & 854 & 884 & 873 & 845 & 835 \\
\hline Average CPU Time & N.A & $2 \mathrm{~h}$ & $2 \mathrm{~h}$ & $4 \mathrm{~h}$ & $3 \mathrm{~h}$ \\
\hline$P_{R}^{p v}(\mathrm{~kW})$ & 500 & 499 & 376 & 500 & 500 \\
\hline$P_{R}^{g e}(\mathrm{~kW})$ & 1,200 & 402 & 1,272 & 1,147 & 1,147 \\
\hline$E_{R}^{b a t}(\mathrm{kWh})$ & 440 & 326 & 31 & 0 & 0 \\
\hline$E_{R}^{t s}(\mathrm{RTh})$ & 190 & 71 & 93 & 8 & 87 \\
\hline$Q_{R}^{c h}(\mathrm{RT})$ & 519 & 609 & 592 & 500 & 370 \\
\hline
\end{tabular}

\section{CONCLUSiONS}

The paper investigates planning strategies for a multi-energy system that supplies both electrical and thermal loads with a set of heterogeneous equipment such as storages units, gas engine coupled with an absorption chiller and solar panels. At first, the work successfully implements a generic linearization to approximate the operating conditions of the gas engine and chiller plant. That MILP formulation reduces the run time to just a few seconds as compared to 5 minutes using the original nonlinear model with SQP. The computation of the energy bill over a representative day is then introduced in an optimal planning methodology that aims at finding the most appropriate capacities of the assets to minimize the yearly TCO of the system. Different approaches are implemented and successive runs display heterogeneous performances. A Monte Carlo simulation based on Latin Hypercube Sampling returns good solution but at the cost of a time-consuming exploration of the search space. Bi-level optimization architectures, and especially the use of a PSO algorithm for the design, significantly shortens the computation while guaranteeing a diversity of the returned solutions. However, the best performances are obtained with an 'all-in-one' approach that consists in a SQP algorithm, which optimizes both the sizing and operating variables at the same time. The method systematically converges toward solutions with lower TCO and displays a good precisions rate (i.e. lower confidence interval with different starting points). More importantly, the computation time is remarkably short, below one minute. Originally, the OPEX is computed over a single representative day but later simulation investigates weekly profiles. The obtained results shows consistency with the original daily simulations with the best solutions (in terms of installed capcities) remaining in the same neighborhood. Finally, the paper consist of a thorough results analysis that can be interpreted as a first step toward robust optimization in order to define the best areas in the search space (in terms of assets capacities) that minimize the objective. The partitioning of the obtained solutions displays a wide range of capacities corresponding to similar values for the yearly TCO.

Regarding potential further works, it should be noted that representing the system environment (i.e. solar radiation profile and load pattern) should be an area of research by itself, involving data analysis to extract the representative patterns for different type of system. For instance, the seasonality effect would depend strongly on the geographical position of the system and if one representative week would be enough in Singapore in the absence of seasonality, more days (or months ?) may be necessary for other locations. The representative period also depends on the nature of the load (e.g. office, residential, commercial, industrial processes, etc). Finally note that for such planning problems, the modeling of the economic environment (i.e. energy prices and policy) has the greatest impact on the obtained results. Thus, the prediction the evolution of prices over the system lifetime should also be carefully studied. That is part of ongoing works and it is not in the scope of the proposed paper. Also, different cost functions should be investigated in addition to the conventional energy arbitrage considered here (e.g. peak shaving, demand response) in order to estimate their impact on the area of 
solutions. A further interesting work would be to integrate the robustness analysis in the formulation of the planning problem itself with the use of stochastic approaches for instance.

\section{AKnowledgement}

This research is supported by the National Research Foundation, Prime Minister's Office, Singapore under its Energy NIC grant (NRF Award No.: NRF-ENIC-SERTD-SMES-NTUJTCI3C-2016) .

\section{REFERENCES}

[1] A.-M. Borbely, J. F. Kreider, "Distributed Generation: The Power Paradigm for the New Millennium", CRC Press, $1^{\text {st }}$ edition, 2001

[2] T. Ackermann, G. Andersson, L. Sode, “Distributed generation: a definition”, Electric Power System Research, Vol57, no 3, pp. $195-204,2001$.

[3] K. Jana, A. Ray, M. Mansouri Majoumerd, M. Assadi, S. De, "Polygeneration as a future sustainable energy solution - A comprehensive review", Applied Energy, Vol 202, pp. 88-111, 2017.

[4] P. Mancarella, "MES (multi-energy systems): An overview of concepts and evaluation models", Energy, Vol 65, pp. 1-17, 2014

[5] A. Rong, R. Lahdelma, "Role of polygeneration in sustainable energy system development challenges and opportunities from optimization viewpoints", Renewable and Sustainable Energy Reviews, Vol 53, pp. 363-372, 2016.

[6] L. Bartolucci, S. Cordiner, V. Mulone, V. Rocco, J.L. Rossi, "Hybrid renewable energy systems for renewable energy integration in microgrids: Influence of sizing on performance", Energy, Vol 152, pp. 744-758, 2018.

[7] T. Ma, J. Wu, L. Hao, W. Lee, H. Yan, D. Li, “The optimal structure planning and energy management strategies of smart multi energy systems”, Energy, Vol 160, pp. 122-141, 2018.

[8] A. Omu, R. Choudhary, A. Boies, “Distributed energy resource system optimisation using mixed integer linear programming”, Energy Policy, Vol 61, pp. 249-266, 2013.

[9] Y. Yang, S. Zhang, Y. Xiao, “An MILP (mixed integer linear programming) model for optimal design of district-scale distributed energy resource systems”, Energy, Vol 90, pp. 1901-1915, 2015.

[10] S. Mashayekh, M. Stadler, G. Cardoso, M. Heleno, "A mixed integer linear programming approach for optimal DER portfolio, sizing, and placement in multienergy microgrids", Applied Energy, Vol 187, pp. 154-168, 2017.

[11] D.F. Dominković, V. Dobravec, Y. Jiang, P.S. Nielsen, G. Krajačić, “Modelling smart energy systems in tropical regions”, Energy, Vol 155, pp. $592-609,2018$.

[12] T. Capuder, P. Mancarella, "Techno-economic and environmental modelling and optimization of flexible distributed multi-generation options", Energy, Vol 71, pp. 516-533, 2014.

[13] L. Li, H. Mu, N. Li, M. Li, "Economic and environmental optimization for distributed energy resource systems coupled with district energy networks", Energy, Vol 109, pp. 947-960, 2016.

[14] H.K. Fathy, J.A. Reyer, P.Y. Papalambos et A.G Ulsoy, “On the coupling between the plant and controller optimization problems", American Control Conference, Arlington, 2001. 
[15] X. Zhang, S. C. Tan, G. Li, J. Li, Z. Feng, "Components sizing of hybrid energy systems via the optimization of power dispatch simulations", Energy, Vol 52, pp. 165-172, 2013.

[16] B. Li, R. Roche, A. Miraoui, “Microgrid sizing with combined evolutionary algorithm and MILP unit commitment”, Applied Energy, Vol 188, pp. 547-562, 2017.

[17] M. H. Moradi, M. Eskandari, H. Showkati, "A hybrid method for simultaneous optimization of DG capacity and operational strategy in microgrids utilizing renewableenergy resources”, Electric Power and Energy System, Vol 56, pp. 241-258, 2014.

[18] R.Rigo-Mariani, B. Sareni and X. Roboam, "Fast power flow scheduling and sensitivity analysis for sizing a microgrid with storage", Mathematics and computers in simulation, Vol 131, pp 114-127, 2017.

[19] M. Quashie, G. Joos, “Optimal Planning of Urban Microgrids with an Energy Management System”, IEEE/PES Transmission and Distribution Conference and Exposition (T\&D), Dallas, USA, 2016.

[20] Y. Han, P. Young, D. Zimmerle, “Optimal selection of generators in a microgrid for fuel usage minimization”, IEEE PES General Meeting, Vancouver, BC, Canada, 2013.

[21] L. Morettia, M. Astolfia, C. Vergarab, E. Macchia, J. I. Pérez-Arriagad, G. Manzolinia, “ A design and dispatch optimization algorithm based on mixed integer linear programming for rural electrification", Applied Energy, pp. 1104-1121, 2019.

[22] E. Merkel, R. McKenna, W. Fichtner, “Optimization of the capacity and the dispatch of decentralised”, Applied Energy, Vol 140, pp. 120-134, 2015.

[23] L. Setyawan, J. Tan, S. Ding, "Large-Scale Modeling and Optimization Strategy for Multi-Energy Management Systems", Asian Conference on Energy, Power and Transportation Electrification (ACEPT), Singapore, 2018.

[24] S. Ooi, S. Mazzoni, A. Romagnoli, "A Microgrid Application of Polygeneration System Fed by Natural Gas: Effect of Fuel Price on Investment Outlook", Asian Conference on Energy, Power and Transportation Electrification (ACEPT), Singapore, 2018.

[25] Mazzoni S., Cerri G., Chennaoui L.: "A Simulation Tool for Concentrated Solar Power based on Micro Gas Turbine Engine”, Energy Conversion and Management Vol 174, pp. 844-854, 2018

[26] A.D. Hawkes, M.A. Leach, "Modelling high level system design and unit commitment for a microgrid", Applied Energy, Vol 86, pp 1253-1265, 2009

[27] K. Van den Bergh, K. Bruninx, E. Delarue, W. D’haeseleer, “A Mixed-Integer Linear Formulation of the Unit Commitment Problem”, KU Leuven, TME working paper, 2014. [Online] Available: https://www.mech.kuleuven.be/en/tme/research/energy_environment/Pdf/wpen2014-07.pdf

[28] M. Carrión, J. M. Arroyo, “A computationally efficient mixed-integer linear formulation for the thermal unit commitment problem”, IEEE Transactions On Power Systems, Vol 21, no. 3, pp 1371-1378, 2006.

[29] C. D'Ambrosio, A. Lodi, S. Martello, "Piecewise linear approximation of functions of two variables in MILP models", Operations Research Letters, Vol 38, pp 3946, 2010.

[30] J. Lofberg, "YALMIP: a toolbox for modelling and optimization in MATLAB", IEEE International Symposium on Computer Aided Control Systems Design, NewOrleans, USA, pp. 282-289, 2004.

[31] M. Rivarolo, A. Cuneo, A. Traverso, A.F. Massardo, "Design optimization of smart poly-generation energy districts through a model based approach", Applied Thermal Engineering, Vol 99, pp 291-301, 2016.

[32] International Renewable Energy Agency, “The Power to Change : Solar and Wind Cost Reduction Potential to 2025”, 2016, https://www.irena.org/publications/2016 
[33] U.S. Department of Energy, "Combined Heat and Power Technology Fact Sheet Series”, 2016, https://www.energy.gov/sites/prod/files/2016/09/f33/CHPMicroturbines_0.pdf

[34] International Renewable Energy Agency, “Electricity Storage and Renewables: Costs and Markets to 2030”, 2017, https://www.irena.org/publications/2017/Oct

[35] M.D. Mckay , R. J. Beckman, W.J. Conover, "A Comparison of Three Methods for Selecting Values of Input Variables in the Analysis of Output From a Computer Code", Technometrics, Vol 42, no 1, pp 55-61, 2012.

[36] J. Agnarsson, M. Sunde, I. Ermilova, "Project in Computational Science: Report", Project Report, Uppsala Universitet, Department of Information Technology, https://www.it.uu.se/edu/course/homepage/projektTDB/ht12/project09/Report 09.pdf 2013

[37] J. Kennedy, R Eberhart, "Particle swarm optimization”, IEEE Proceedings of the international conference on neural networks, pp. 1942-1948, 1995.

[38] J. Fiala, B. Marteau, "Nonlinear Optimization: A Comparison of Two Competing Approaches", NAG publications, 2017. https://www.nag.co.uk/market/nonlinearoptimization-comparison.pdf

[39] Mazzoni S, Ooi S, Nastasi B, Romagnoli A. Energy storage technologies as techno-economic parameters for master-planning and optimal dispatch in smart multi energy systems. Applied Energy 2019;254:113682. doi:10.1016/j.apenergy.2019.113682. 